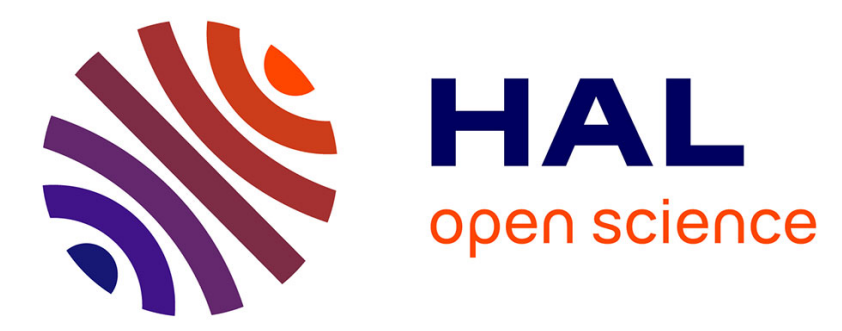

\title{
A GENERATING FUNCTION FOR RANKIN-COHEN BRACKETS
}

\author{
Michael Pevzner
}

\section{To cite this version:}

Michael Pevzner. A GENERATING FUNCTION FOR RANKIN-COHEN BRACKETS. Letters in Mathematical Physics, 2018, 10.1007/s11005-018-1085-9 . hal-01806455

\section{HAL Id: hal-01806455 \\ https://hal.science/hal-01806455}

Submitted on 11 Jun 2018

HAL is a multi-disciplinary open access archive for the deposit and dissemination of scientific research documents, whether they are published or not. The documents may come from teaching and research institutions in France or abroad, or from public or private research centers.
L'archive ouverte pluridisciplinaire HAL, est destinée au dépôt et à la diffusion de documents scientifiques de niveau recherche, publiés ou non, émanant des établissements d'enseignement et de recherche français ou étrangers, des laboratoires publics ou privés. 


\title{
A GENERATING FUNCTION FOR RANKIN-COHEN BRACKETS
}

\author{
MICHAEL PEVZNER
}

\begin{abstract}
We give a closed formula for the Rankin-Cohen formal covariant quantization in terms of generating functions of Jacobi polynomials.
\end{abstract}

The idea to interpret the quantization procedure as a formal deformation of the commutative algebra structure of classical observables comes back to the celebrated work of F. Bayen, M. Flato, C. Fronsdal, A. Lichnerowicz and D. Sternheimer (2, 3]). Apart from physical considerations motivated by the perturbation theory this approach is originated from the semi-classical analysis of the Weyl operator calculus which is a way to associate with a reasonable function, say $f \in L^{2}\left(\mathbb{R}^{2 n}\right)$, an operator $\mathrm{Op}_{W}(f)$ defined on $L^{2}\left(\mathbb{R}^{n}\right)$ by

$$
\left(\mathrm{Op}_{W}(f) u\right)(x)=\hbar^{-n} \iint_{\mathbb{R}^{n} \times \mathbb{R}^{n}} f\left(\frac{x+y}{2}, \eta\right) e^{\frac{2 i \pi}{h}\langle x-y, \eta\rangle} u(y) d y d \eta \quad u \in L^{2}\left(\mathbb{R}^{n}\right) .
$$

Notice that the composition of two operators $\mathrm{Op}_{W}\left(f_{1}\right) \circ \mathrm{Op}_{W}\left(f_{2}\right)$ is given in terms of their Weyl symbols $f_{1}$ and $f_{2}$ (without loss of generality one may assume $f_{1}, f_{2} \epsilon$ $\left.\mathcal{S}\left(\mathbb{R}^{2 n}\right)\right)$ as follows:

$$
\begin{aligned}
f_{1} \star f_{2}(X) & =\mathrm{Op}_{W}^{-1}\left(\mathrm{Op}_{W}\left(f_{1}\right) \circ \mathrm{Op}_{W}\left(f_{2}\right)\right) \\
& =(2 / \hbar)^{2 n} \int_{\mathbb{R}^{2 n} \times \mathbb{R}^{2 n}} f_{1}(Y) f_{2}(Z) e^{-4 i \pi \hbar^{-1} \omega(Y-X, Z-X)} d Y d Z
\end{aligned}
$$

Using spectral theory we get another, fully equivalent, expression for the composition of symbols

$$
f_{1} \star f_{2}(X)=\operatorname{Rest}_{\mid X=Y=Z}, \exp (i \pi L)\left(f_{1}(Y) \cdot f_{2}(Z)\right) .
$$

where $X=(x, \eta), Y=(y, \eta), Z=(z, \zeta) \in \mathbb{R}^{2 n}, \omega(X, Y)=-\langle x, \eta\rangle+\langle y, \xi\rangle$ is the standard symplectic form on $\mathbb{R}^{2 n}$ and

$$
i \pi L=\frac{\hbar}{4 i \pi} \sum_{j=1}^{n}\left(-\frac{\partial^{2}}{\partial y_{j} \partial \zeta_{j}}+\frac{\partial^{2}}{\partial z_{j} \partial \eta_{j}}\right) .
$$

2010 Mathematics Subject Classification. 53D55. 
MICHAEL PEVZNER

Expanding the exponential into a series, the expression (4) gives rise to the Moyal formula

$$
\begin{aligned}
& \left(f_{1} \star f_{2}\right)(x, \xi) \\
& =\sum \frac{(-1)^{|\alpha|}}{\alpha ! \beta !}\left(\frac{\hbar}{4 i \pi}\right)^{|\alpha|+|\beta|}\left(\frac{\partial}{\partial x}\right)^{\alpha}\left(\frac{\partial}{\partial \xi}\right)^{\beta} f_{1}(x, \xi)\left(\frac{\partial}{\partial x}\right)^{\beta}\left(\frac{\partial}{\partial \xi}\right)^{\alpha} f_{2}(x, \xi)
\end{aligned}
$$

which can be understood as an asymptotic expansion of $\left(f_{1} \star f_{2}\right)$ with respect to the rescaling parameter $\hbar$ and yields the prototypical example of a deformation quantization or a $\star$-product.

The exponential function arising in the expression (4) plays an important role in the whole construction and underlines the very nature of the Weyl calculus which can be understood as an extension of the Schrödinger representation of the Heisenberg Lie algebra $\mathfrak{h}_{n}$ to the group algebra of the Heisenberg group.

Both approaches, the operator calculus and the formal deformation have their own interests and merits. The aim of this short note is to give an explicit formula analogous to (4) for the covariant deformation quantization of the upper half-plane based on the family of Rankin-Cohen bi-differential operators.

We notice that the first two terms of (6) are respectively the usual point-wise product of functions $f_{1} \cdot f_{2}$ and their image $\left\{f_{1}, f_{2}\right\}$ by the Poisson bracket associated with the standard (constant coefficient) symplectic form $\omega(\cdot, \cdot)$ on $\mathbb{R}^{2 n}$.

The fact that any smooth Poisson structure on a real manifold $M$ defines a formal associative product on $C^{\infty}(M)$ is a deep result of cohomological nature that follows from Kontsevich' formality theorem. In presence of symmetries, that is, under the assumption that the manifold $M$ is a homogeneous space, the construction of a covariant deformation is even more involved and different techniques were recently developed for appropriate classes of symmetry groups (see for instance [5, 11, 12, 13, [18, 19] and the literature quoted therein).

One such a nontrivial but handable example is provided by the covariant quantization of the Lobachevsky upper half plane $H_{2}=\{z=x+i y \in \mathbb{C}: y>0\} \simeq$ $S L(2, \mathbb{R}) / S O(2, \mathbb{R})$. One may mention at least four different constructions. Namely,

(1) A. Unterberger and J. Unterberger ( $c f$. [21]) developed an $S L(2, \mathbb{R})$-equivariant analog of the Weyl operator calculus which introduces, in the spirit of (2), a covariant noncommutative product of functions on $\mathrm{H}_{2}$ via the composition of operators they correspond to.

It turns out that in an appropriate setting such a product can be expressed in terms of Rankin-Cohen brackets defined for any $a \in \mathbb{N}$ and $f_{j} \in B^{k_{j}}\left(H_{2}\right):=$ 
$\mathcal{O}\left(H_{2}\right) \cap L^{2}\left(H_{2}, y^{k_{j}-2} d x d y\right)$, with $k_{j} \in \mathbb{N} \backslash\{0\}, j=1,2$ by:

$\mathcal{R} \mathcal{C}_{k_{1}, k_{2}}^{k_{3}}\left(f_{1}, f_{2}\right)(z):=\sum_{\ell=0}^{a}(-1)^{\ell}\left(\begin{array}{c}k_{1}+a-1 \\ \ell\end{array}\right)\left(\begin{array}{c}k_{2}+a-1 \\ a-\ell\end{array}\right) f_{1}^{(a-\ell)}(z) f_{2}^{(\ell)}(z)$,

where $f^{(n)}(z)=\frac{d^{n}}{d z^{n}} f(z)$, and $k_{3}:=k_{1}+k_{2}+2 a$ (see [6]).

More precisely, the associativity of the natural composition of operators implies that the formula

$$
f_{1 \sharp} f_{2}=\sum_{a \in \mathbb{N}} c_{k_{1}, k_{2}}^{k_{3}} \mathcal{R C}_{k_{1}, k_{2}}^{k_{1}+k_{2}+2 a}\left(f_{1}, f_{2}\right),
$$

defined for $f_{j} \in B^{k_{j}}\left(H_{2}\right), k_{j} \in \mathbb{N} \backslash\{0\}, j=1,2$, where $c_{k_{1}, k_{2}}^{k_{3}}$ are explicit constants obtained from the multiplication table of reproducing kernels of Hilbert spaces $B^{k_{j}}\left(H_{2}\right), k_{j} \in \mathbb{N} \backslash\{0\}$ for $j=1,2,3$ (see [21, Theorem 4.2]), gives rise to an associative, noncommutative product on $\mathrm{H}_{2}$ deforming the point-wise product $\mathcal{R C}_{k_{1}, k_{2}}^{k_{1}+k_{2}}$ in the direction of the Poisson bracket $\mathcal{R C}_{k_{1}, k_{2}}^{k_{1}+k_{2}+2}$.

(2) P. Cohen, Yu. Manin and D. Zagier (cf. [7]) followed the opposite way and having imposed de facto the associativity constraint on formal expressions of the form

$$
f_{1} \star_{\alpha} f_{2}:=\sum_{a \in \mathbb{N}} \hbar^{a} \alpha_{k_{1}, k_{2}}^{k_{3}} \mathcal{R} \mathcal{C}_{k_{1}, k_{2}}^{k_{1}+k_{2}+2 a}\left(f_{1}, f_{2}\right)
$$

found, up to a rescaling of coefficients $\alpha_{k_{1}, k_{2}}^{k_{3}}$, the same expression for the deformed product as in (8).

The fact that the authors looked for formal deformations given in terms of Rankin-Cohen brackets is not an ad hoc requirement: one shows (e.g. [10]) that such bi-differential operators are the only, up to a scalar multiple, operators intertwining the tensor product of $S L(2, \mathbb{R})$ representations in $B^{k_{1}}\left(H_{2}\right)$ and $B^{k_{2}}\left(H_{2}\right)$ with its irreducible component isomorphic to $B^{k_{3}}\left(H_{2}\right)$ (such representations are precisely the holomorphic discrete series representations of the Lie group $S L(2, \mathbb{R})$ ).

Motivated by the work [7] A. Connes and H. Moscovici gave an intrinsic explanation of the associativity of deformations of type (8) in terms of Hopf algebras of transversal geometry ([8]). The authors also emphasized that putting all the combinatorial coefficients $\alpha_{k_{1}, k_{2}}^{k_{3}}$ to be equal to one leads, as predicted by W. Eholzer and T. Ibukiyama ( $c f[9])$, to an associative product:

$$
f_{1} \star_{1} f_{2}:=\sum_{a \in \mathbb{N}} \hbar^{a} \mathcal{R} \mathcal{C}_{k_{1}, k_{2}}^{k_{1}+k_{2}+2 a}\left(f_{1}, f_{2}\right) \text {. }
$$

(3) One may also capture the associativity of a formal deformation through the Yang-Baxter equation and, in the covariant setting, the fusion matrices for 
Verma modules. This construction is due to A. Alekseev and A. Lachowska [1] and the link with Rankin-Cohen deformations (8) was established by M. Medina-Luna in [17].

(4) Yet another method is based on the Fedosov construction adapted by P. Bieliavsky, X. Tang and Y. Yao [4] to the natural symplectic structure of $\mathrm{H}_{2}$.

However none of these methods exhibits a closed formula of type (4) for the covariant quantization of the Lobachevsky upper half-plane $H_{2}$.

Investigating the general structure of intertwining operators for discretely decomposable branching rules of infinite dimensional representations of real reductive Lie groups (the decomposition $B^{k_{1}}\left(H_{2}\right) \otimes B^{k_{2}}\left(H_{2}\right) \simeq \widehat{\oplus}_{a \in \mathbb{N}} B^{k_{1}+k_{2}+2 a}\left(H_{2}\right)$ into irreducible components of the tensor product of two holomorphic discrete series representations of $S L(2, \mathbb{R})$ mentioned above is a first example of this paradigm) it was recently pointed out ( $c f$ [16, (9.9)]) that Rankin-Cohen brackets are related to the classical Jacobi polynomials:

$$
\begin{aligned}
P_{\ell}^{\alpha, \beta}(t) & =\frac{\Gamma(\alpha+\ell+1)}{\Gamma(\alpha+1) \ell !}{ }_{2} F_{1}\left(-\ell, \alpha+\beta+\ell+1 ; \alpha+1 ; \frac{1-t}{2}\right) \\
& =\frac{\Gamma(\alpha+\ell+1)}{\Gamma(\alpha+\beta+\ell+1)} \sum_{m=0}^{\ell}\left(\begin{array}{c}
\ell \\
m
\end{array}\right) \frac{\Gamma(\alpha+\beta+\ell+m+1)}{\Gamma(\alpha+m+1) \ell !}\left(\frac{t-1}{2}\right)^{m},
\end{aligned}
$$

where ${ }_{2} F_{1}(a, b ; c ; z)$ denotes the Gauss hypergeometric function that can be defined, for instance, for $z \in \mathbb{C}:|z|<1$ by a power series $=\sum_{n \in \mathbb{N}} \frac{(a)_{n}(b)_{n}}{(c)_{n} n !} z^{n}$ (here $(x)_{n}$ denotes the rising Pochhammer symbol).

Namely, define a homogeneous polynomial in two variables obtained from the classical Jacobi polynomial $P_{\ell}^{\alpha, \beta}(t)$ of degree $\ell$ by the following inflation procedure:

$$
\begin{aligned}
P_{\ell}^{\alpha, \beta}(x, y) & :=y^{\ell} P_{\ell}^{\alpha, \beta}\left(2 \frac{x}{y}+1\right) \\
& =\frac{\Gamma(\alpha+\ell+1)}{\Gamma(\alpha+\beta+\ell+1)} \sum_{m=0}^{\ell}\left(\begin{array}{c}
\ell \\
m
\end{array}\right) \frac{\Gamma(\alpha+\beta+\ell+m+1)}{\Gamma(\alpha+m+1) \ell !} x^{m} y^{\ell-m} .
\end{aligned}
$$

Then, for avery $a \in \mathbb{N}$ the following identity on holomorphic bidifferential operators holds:

$$
\mathcal{R} \mathcal{C}_{k_{1}, k_{2}}^{k_{1}+k_{2}+2 a}=\left.\operatorname{Rest}\right|_{z_{1}=z_{2}=z} \circ(-1)^{a} P_{a}^{k_{1}-1,1-k_{1}-k_{2}-2 a}\left(\frac{\partial}{\partial z_{1}}, \frac{\partial}{\partial z_{2}}\right) .
$$

Of course, once known, the above formula (13) is elementary to check. The general method, referred to as the $F$-method and developed in [15], gives a general framework for constructing all differential symmetry breaking operators for discretely decomposable branching rules of real reductive Lie groups and explains the "raison d'être" 
of the formula (13) interpreting the covariance property of the Rankin-Cohen bidifferential operators $\mathcal{R C}_{k_{1}, k_{2}}^{k_{3}}$ as a constraint on their symbols, a constraint which turns out to be in this case the Gauss hypergeometric equation.

The formula 13 can be expressed in a more symmetric way.

Lemma 1. Let $\widetilde{P}_{\ell}^{\alpha, \beta}(x, y)=(-1)^{\ell}(x+y)^{\ell} P_{\ell}^{\alpha, \beta}\left(\frac{y-x}{x+y}\right)$. Then, for any $a \in \mathbb{N}$ :

$$
\mathcal{R C}_{k_{1}, k_{2}}^{k_{1}+k_{2}+2 a}=\left.\operatorname{Rest}\right|_{z_{1}=z_{2}=z} \widetilde{P}_{a}^{k_{1}-1, k_{2}-1}\left(\frac{\partial}{\partial z_{1}}, \frac{\partial}{\partial z_{2}}\right)
$$

Proof. According to the first Kummer's relation for the hypergeometric function (see for instance [14, 8.962]):

$$
\begin{aligned}
P_{\ell}^{\alpha, \beta}(t) & =\frac{(-1)^{\ell} \Gamma(\ell+1+\beta)}{\ell ! \Gamma(1+\beta)}{ }_{2} F_{1}\left(\ell+\alpha+\beta+1,-\ell ; 1+\beta ; \frac{1+t}{2}\right) \\
& =\frac{\Gamma(\ell+1+\beta)}{\ell ! \Gamma(1+\beta)}\left(\frac{t-1}{2}\right)_{2}^{\ell} F_{1}\left(-\ell,-\ell-\alpha ; 1+\beta ; \frac{1+t}{t-1}\right) \\
& =\frac{\Gamma(\ell+1+\alpha)}{\ell ! \Gamma(1+\alpha)}\left(\frac{t+1}{2}\right)_{2}^{\ell} F_{1}\left(-\ell,-\ell-\beta ; 1+\alpha ; \frac{t-1}{t+1}\right)
\end{aligned}
$$

we get

and therefore

$$
P_{\ell}^{\alpha, \beta}(t)=\left(\frac{1+t}{2}\right)^{\ell} P_{\ell}^{\alpha,-\alpha-\beta-2 \ell-1}\left(\frac{3-t}{1+t}\right)
$$

$$
P_{\ell}^{k_{1}-1,1-k_{1}-k_{2}-2 a}(1-2 s)=(1-s)^{\ell} P_{\ell}^{k_{1}-1, k_{2}-1}\left(\frac{1+s}{1-s}\right)
$$

what concludes the proof together with the definition (12).

Let us recall $((c f[20,(4.4 .5)])$ that the generating series of Jacobi polynomials is given by :

$$
\sum_{\ell \geq 0} P_{\ell}^{\alpha, \beta}(t) z^{\ell}=2^{\alpha+\beta} R^{-1}(1-z+R)^{-\alpha}(1+z+R)^{-\beta},
$$

where $R=r(t, z)=\sqrt{1-2 t z+z^{2}}$. This leads us to the following conclusion.

Theorem 2. Consider the following function of two variables $(x, y)$ depending on a formal parameter $\hbar$ :

$$
\begin{aligned}
& F_{\alpha, \beta}^{\hbar}(x, y)= \\
& \frac{\left(1+\hbar(x+y)+\sqrt{1+2(y-x) \hbar+\hbar^{2}(x+y)^{2}}\right)^{-\alpha}\left(1-\hbar(x+y)+\sqrt{1+2(y-x) \hbar+\hbar^{2}(x+y)^{2}}\right)^{-\beta}}{2^{-\alpha-\beta} \sqrt{1+2(y-x) \hbar+\hbar^{2}(x+y)^{2}}}
\end{aligned}
$$


Then the formal Rankin-Cohen covariant quantization ${ }_{1}$ of the upper half-plane $\mathrm{H}_{2}$ is given by

$$
\begin{aligned}
f_{1} \star_{1} f_{2}(z) & =\sum_{a \in \mathbb{N}} \hbar^{a} \mathcal{R C}_{k_{1}, k_{2}}^{k_{1}+k_{2}+2 a}\left(f_{1}, f_{2}\right) \\
& =\left.\operatorname{Rest}\right|_{x=y=z} \circ F_{k_{1}, k_{2}}^{\hbar}\left(\frac{\partial}{\partial x}, \frac{\partial}{\partial y}\right)\left(f_{1}(x) \cdot f_{2}(y)\right),
\end{aligned}
$$

where $f_{k_{j}} \in B^{k_{j}}\left(H_{2}\right), k_{j} \in \mathbb{N} \backslash\{0\}, j=1,2$.

Proof. The formula (14) for the generating function of Jacobi polynomials implies that

$$
\sum_{\ell \geq 0}(-1)^{\ell} P_{\ell}^{\alpha, \beta}\left(\frac{y-x}{x+y}\right)(x+y)^{\ell} \hbar^{\ell}=F_{\alpha, \beta}^{\hbar}(x, y) .
$$

Together with Lemma 1 this observation concludes the proof.

\section{REFERENCES}

[1] A. Alekseev,A. Lachowska, Invariant *-products on coadjoint orbits and the Shapovalov pairing. Comment. Math. Helv. 80 (2005), pp. 795-810.

[2] F. Bayen, M. Flato, C. Fronsdal, A. Lichnerowicz, D. Sternheimer, Deformation theory and quantization. I. Deformations of symplectic structures. Ann. Physics 111 (1978), pp. 61-110.

[3] F. Bayen, M. Flato, C. Fronsdal, A. Lichnerowicz, D. Sternheimer, Deformation theory and quantization. II. Physical applications. Ann. Physics 111 (1978), pp. 111-151.

[4] P. Bieliavsky, X. Tang, Y. Yao, Rankin-Cohen brackets and formal quantization. Adv. Math. 212 (2007), pp. 293-314.

[5] P. Bieliavsky, V. Gayral, Deformation quantization for actions of Kählerian Lie groups. Mem. Amer. Math. Soc. 236 (2015), no. 1115, vi+154 pp.

[6] H. Cohen, Sums involving the values at negative integers of $L$-functions of quadratic characters, Math. Ann. 217 (1975), pp. 271-285.

[7] P. B. Cohen, Yu. I. Manin, D. Zagier, Automorphic pseudodifferential operators. Automorphic pseudodifferential operators. In Algebraic aspects of integrable systems, pp. 17-47, Progr. Nonlinear Differential Equations Appl., 26, Birkhäuser Boston, 1997.

[8] A. Connes, H. Moscovici, Rankin-Cohen brackets and the Hopf algebra of transverse geometry, Mosc. Math. J. 4, No.1,(2004), pp. 111-130.

[9] W. Eholzer, T. Ibukiyama, Rankin-Cohen type differential operators for Siegel modular forms. Int. J. Math. 9, no. 4 (1998), pp. 443-463.

[10] G. van Dijk, M. Pevzner, Ring structures for holomorphic discrete series and Rankin-Cohen brackets. J. Lie Theory, 17, (2007), pp. 283-305.

[11] V. Dolgushev, Covariant and equivariant formality theorems. Adv. Math. 191 (2005), pp. 147177.

[12] V. Fischer, M. Ruzhansky, Quantization on nilpotent Lie groups. Progress in Mathematics, 314. Birkhäuser-Springer, 2016. xiii+557 pp.

[13] V. Gayral, D. Jondreville, Deformation quantization for actions of $\mathbb{Q}_{p}^{d}$. J. Funct. Anal. 268 (2015), pp. 3357-3403. 
[14] I. S. Gradshteyn, I. M. Ryzhik. Table of integrals, series, and products.Translated from the Russian. Translation edited and with a preface by Daniel Zwillinger and Victor Moll. Eighth edition. Revised from the seventh edition. Elsevier/Academic Press, Amsterdam, 2015. xlvi+1133 pp.

[15] T. Kobayashi, M. Pevzner, Differential symmetry breaking operators. I. General theory and F-method. Selecta Math.(N.S.) 22, 801-845,

[16] T. Kobayashi, M. Pevzner, Differential symmetry breaking operators. II. Rankin-Cohen operators for symmetric pairs. Selecta Math. (N.S.) 22, 847-911 (2016).

[17] M. Medina-Luna, Opérateurs de Rankin-Cohen et matrices de fusion, Thèse de doctorat. Université de Reims Champagne-Ardenne, (2016). 136pp.

[18] M. Pevzner, Covariant quantization: spectral analysis versus deformation theory. Jpn. J. Math. 3 (2008), pp. 247-290.

[19] M. Pevzner, Rankin-Cohen brackets and representations of conformal Lie groups. Ann. Math. Blaise Pascal 19 (2012), pp. 455-484.

[20] G. Szegö, Orthogonal Polynomials, A.M.S. Colloquium Publications, Vol. XXIII, Providence, Rhode Island, (1939).

[21] A. Unterberger, J. Unterberger, Algebras of symbols and modular forms. J. Anal. Math. 68, (1996), pp. 121-143.

M. Pevzner. Laboratoire de Mathématiques de Reims, Fre 2011, Université de Reims Champagne-Ardenne, F-51687, Reims, France \& ISC J.-V. Poncelet, CNRS UMI 2615, PEVZNER@UNIV-REIMS.FR. 